\title{
A Multi-Modal Approach for Driver Gaze Prediction to Remove Identity Bias
}

\author{
Zehui Yu \\ PRIS Lab, Beijing University of Posts \\ and Telecommunications \\ DiDi Chuxing \\ Beijing, China \\ yuzehui_i@didiglobal.com \\ Haifeng Shen \\ DiDi Chuxing \\ Beijing, China \\ shenhaifeng@didiglobal.com
}

\author{
Jian Tang \\ DiDi Chuxing \\ Beijing, China \\ tangjian@didiglobal.com
}

\author{
Xiehe Huang \\ PRIS Lab, Beijing University of Posts \\ and Telecommunications \\ Beijing, China \\ Xiehe.Huang@bupt.edu.cn
}

\author{
Qun Li \\ DiDi Chuxing \\ Beijing, China \\ liquntracy@didiglobal.com
}

\author{
Yi Yang \\ DiDi Chuxing \\ Beijing, China \\ yangyiian@didiglobal.com
}

\author{
Xiubao Zhang \\ DiDi Chuxing \\ Beijing, China \\ zhangxiubao@didiglobal.com
}

\begin{abstract}
Driver gaze prediction is an important task in Advanced Driver Assistance System (ADAS). Although the Convolutional Neural Network $(\mathrm{CNN})$ can greatly improve the recognition ability, there are still several unsolved problems due to the challenge of illumination, pose and camera placement. To solve these difficulties, we propose an effective multi-model fusion method for driver gaze estimation. Rich appearance representations, i.e. holistic and eyes regions, and geometric representations, i.e. landmarks and Delaunay angles, are separately learned to predict the gaze, followed by a score-level fusion system. Moreover, pseudo-3D appearance supervision and identity-adaptive geometric normalization are proposed to further enhance the prediction accuracy. Finally, the proposed method achieves state-of-the-art accuracy of $82.5288 \%$ on the test data, which ranks 1st at the EmotiW2020 driver gaze prediction sub-challenge.
\end{abstract}

\section{CCS CONCEPTS}

- Computing methodologies $\rightarrow$ Activity recognition and understanding; Appearance and texture representations.

\section{KEYWORDS}

Driver Gaze Prediction; Appearance Representation; Geometric Representation; Model Fusion; Identity Bias

Permission to make digital or hard copies of all or part of this work for personal or classroom use is granted without fee provided that copies are not made or distributed for profit or commercial advantage and that copies bear this notice and the full citation on the first page. Copyrights for components of this work owned by others than the author(s) must be honored. Abstracting with credit is permitted. To copy otherwise, or republish, to post on servers or to redistribute to lists, requires prior specific permission and/or a fee. Request permissions from permissions@acm.org.

ICMI '20, October 25-29, 2020, Virtual event, Netherlands

(c) 2020 Copyright held by the owner/author(s). Publication rights licensed to ACM. ACM ISBN 978-1-4503-7581-8/20/10 . \$ \$15.00

https://doi.org/10.1145/3382507.3417961
ACM Reference Format:

Zehui Yu, Xiehe Huang, Xiubao Zhang, Haifeng Shen, Qun Li, Weihong Deng, Jian Tang, Yi Yang, and Jieping Ye. 2020. A Multi-Modal Approach for Driver Gaze Prediction to Remove Identity Bias. In Proceedings of the 2020 International Conference on Multimodal Interaction (ICMI '20), October 25-29, 2020, Virtual event, Netherlands. ACM, New York, NY, USA, 9 pages. https://doi.org/10.1145/3382507.3417961

\section{INTRODUCTION}

According to a World Health Organization report, there are more than 1 million people perishing in traffic accidents every year and this trend is still growing year by year. Among many other solutions, Advanced Driver Assistance System (ADAS) is fundamental to prevent these tragedies from happening. The ADAS can monitor the mental status of the driver and therefore, makes driving safe and sound. Driver Gaze Estimation plays a key role in ADAS as it can remind the driver not to be distracted.

For the EmotiW competition, Driver Gaze Prediction challenge[4] was first held in 2020. The task here is basically to predict which zone of a car that the driver is looking at. There are nine zones to be classified, e.g. back mirror, side mirrors, radio, speedometer, and windshield. The data has been collected in Hyundai car with different subjects at the driver position. Stickers with numbers on them are pasted in nine zones respectively, and those volunteers are asked to look at these zones one by one. The whole process is recorded by the Microsoft Lifecam RGB camera. There is no constraint to the subjects about looking by eye movements or head movements, which results a more realistic environment for driving. Furthermore, some subjects may wear glasses or sunglasses and it may cause reflective phenomenon which can reduce the accuracy of gaze prediction seriously. The dataset is collected during different times of the day in order to increase the variance of illumination. It is worth mentioning that those drivers are told to speak out the number on a certain sticker when they gaze at the sticker and the 


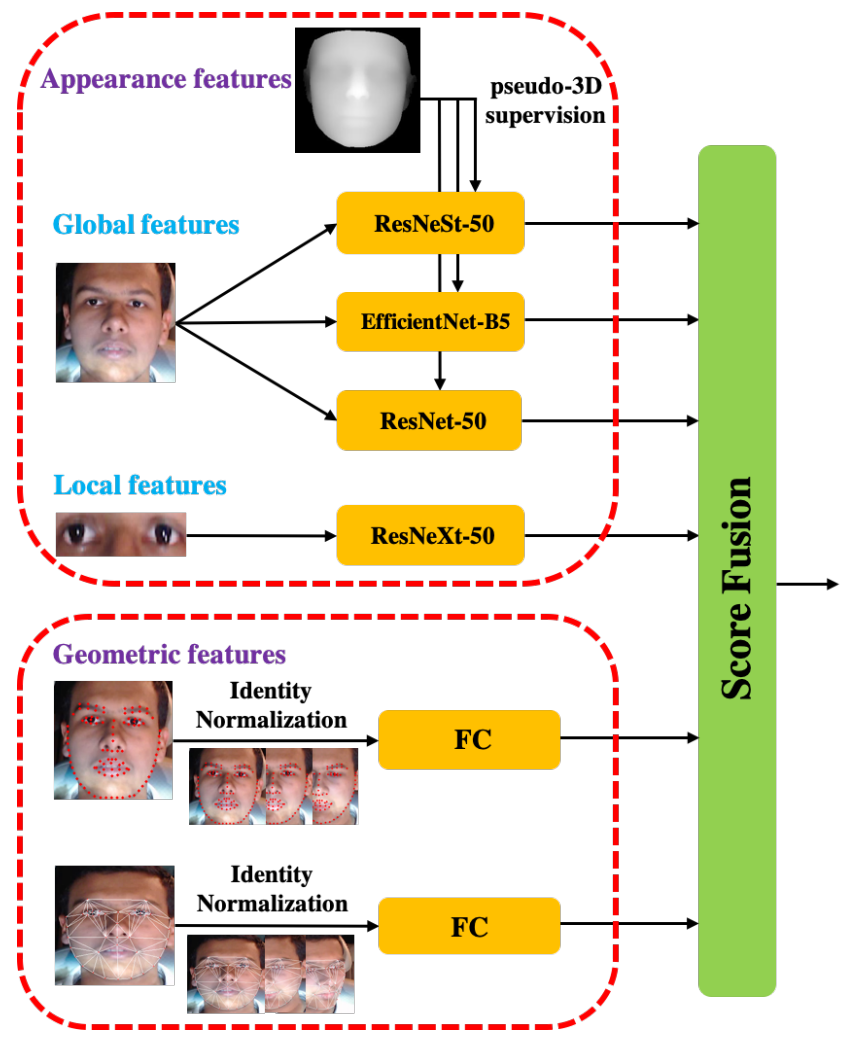

Figure 1: The overview of our proposed framework.

voice will be recorded by a microphone. This Driver Gaze in the Wild (DGW) dataset [7] is the largest publicly available diver gaze estimation dataset as of today and the labels can be generated in an automatic manner using speech-to-text conversion.

As with many other gaze estimation datasets, DGW's data within different splits, i.e. training set, validation set, and test test, are subject-exclusive, such that developers of gaze estimation algorithm are expected to devise a more generalized model. Therefore, identity bias, that is, features related to personal identity, should be reduced during training of a model as much as possible, in order to make the model more generalized.

In our work, we propose to handle the driver gaze prediction task in a multi-modal fashion, that is, taking into consideration not only appearance-based features but also geometrical and 3dimensional (3D) ones. Such features used in our work include face images and eye images for the appearance based part, and facial landmarks and angular degrees from Delaunay triangulation for the geometrical part. The choosing of these features are all for bias-reducing purpose.

First of all, an initial attempt is made to reduce person-dependent bias by training a separate model using only eye-region patches. To greatly minimize the influence of identity bias, we intentionally leave out all the features on a face except eye region. By doing so, a model is forced to extract features from eye area only, instead of all identity-related regions such as outer contour of a face, nose, mouth, and so on.
Furthermore, 3-dimensional information should not be ignored when trying to grapple with person-specific bias. In our case, we adopt UV position maps generated by PRNet [5] as our 3D information. On the one hand, these position maps, as a supervision signal, force our model to learn head pose information implicitly, so that several candidate zones can be picked out for final prediction. On the other hand, the 3D supervision can eliminate identity bias and warn the model not to focus too much on learning facial features for face recognition. As a result, the trained model can learn to exploit more spatial representation and gets a better performance on the cross-subject testing.

Finally, we also include person-independent geometrical features when training individual models. In our work, facial landmarks give our model an insight into both the head pose of a particular sample and the relative position of pupils with respect to, for example, the inner corners and outer corners of eyes. Both of them are conducive to a better prediction of drive gaze. In addition, angular degree from Delaunay triangulation also provides our model with the relative position of eyeball but in a angular way. This means it is more forthright because, as can be seen in Fig. 5 , movement of eyeball can cause all those angles in the triangles of eye area to change unanimously. When training the two models, we adopt an identity normalization method where each input vector will subtract the corresponding ID's average landmark or Delaunay angles for pre-processing. From the experimental results, this identity-based normalization can greatly improve the cross-id accuracy in gaze estimation.

Use those aforementioned features respectively to train a model for each of them, and we will be able to implement a model ensemble to produce the final prediction. The reason why we tackle the task in such way is because we believe one feature among our proposed features is independent from the others and thus every model trained on each feature can provide a unique 'perspective' to our final decision. When every independent feature is exploited, our classifier is supposed to be more likely to make a sensible prediction.

To summarize, our contributions include:

- propose a multi-modal method to grapple with gaze estimation task so as to better predict gaze direction using independent features;

- supervise some layers of a network with depth map so as to improve accuracy on cross-subject data;

- adopt normalized geometrical structures to estimate gaze direction and prove it to be essential when dealing with this task.

The remaining part of the paper proceeds as follows. Some related works will be discussed in section 2 . In section 3 , we will explain our main networks in the proposed framework, including the facial model, the eyes model, the landmark model and the Delaunay model. The experiment results on the challenge dataset will be demonstrated on section 4 . In the end, we will conclude our fusion method in section 5 .

\section{RELATED WORK}

Driver gaze prediction has been a popular research topics in driving monitoring. A prominent approach for gaze zone estimation is remote eye tracking. However, due to the high requirements 
on hardware and the inconvenience in the driving environment, vision based systems appear to be a more attractive solution for the driver gaze prediction task. A number of researchers have reported different vision-based methods in this challenge. Tawari et al. [16] combined static head pose with temporal dynamics to classify the driver zones. Vasli et al. [18] further make a fusion of head pose and eyes features and get a quite impressive result. However, these features are mainly based on handcraft and can't adapt to the large variance on different subjects or pose.

With the deep learning development, the features extracted by $\mathrm{CNN}$ show more powerful representation and generalization capabilities than the features designed by handcraft. Naqvi et al. [12] combine head pose estimation and pupil detection to predict the gaze zone, but the datasets are collected in a relatively ideal condition and cannot apply to the real driving environment. Jah et al. [11] use head pose to estimate the direction, but head pose features may fail to discriminate between adjacent zones which can separated by subtle eye movement. In our framework, we fusion three kinds of features from three state-of-the-art CNNs, i.e. ResNeSt-50, EfficientNet-B5 and ResNet-50, which can improve the representation ability of features as much as possible. Simultaneously, 3D supervision is used to help the model learn head pose features implicitly for better generalization on different subjects.

The area of gaze estimation, for the past decades, have been mostly focusing on regression-based methods as researchers tend to devise a more generalized model. Following this line of thought, Zhang et al. [23] employ appearnce-based multi-modal convolutional neural networks and apply space normalization technique to normalize all the data. They also present a large-scale dataset named MPIIGaze for researchers around the world. Chen et al. [1] decompose the task into subject-independent estimator and subjectdependent calibrator in order to solve individual variations. In our work, we make a few modifications to Chen's Gaze Decomposition when experimenting with eye images. First, we replace all the branches featured by dilated convolutions with ResNeXt-50 [20], which is proven in our experiments to be more effective. Secondly, we add yet another branch which takes two-eye images as input, so as to learn holistic features from two eyes as a whole instead of separate eye. Thirdly, we further concatenate those features extracted from three branches with normalized eye landmark coordinates.

An obvious trend in the gaze estimation community is that researchers are more and more predisposed towards using geometrical information to facilitate predicting of gaze direction. Among those frequently used geometrical data is facial landmarks, each of which indicates a specific location on a face, e.g. pupils, mouth corners, and nose tip. Notice that we adopt the method proposed by Huang et al. [10] to produce facial landmark coordinates for samples in DGW dataset. Another geometrical feature is angles obtained by Delaunay triangulation, a way to triangulate facial keypoints on a face. In Fridman et al. [6] work, they extract landmarks from the faces and design a Delaunay triangulation over the 19 landmarks. The three angles of each of the resulting triangles are added to the landmark vectors and the fusion vectors are used to generate class probabilities by a random forest classifier.

\section{PROPOSED METHOD}

Our proposed framework mainly consists of two parts, i.e. appearancebased features and geometry-based features. As for the appearance features, we fusion three mainstream $\mathrm{CNN}$ networks, i.e. ResNet50[9], ResNeSt-50[21] and EfficientNet[15] to extract global facial features. The eyes image model, for the local features, is similar to the facial image model, but we use ResNeXt-50[20] as the backbone and the input contains faces, double eyes, single eye and normalized landmarks. Considering the importance of geometrical representation, we introduce the landmark model and Delaunay model to enrich the feature extraction. All the predictions from the above models are weighted with a gird-search optimization and the fusion predictions will be used for the final classification. Because of the person-independent task, some tricks, such as 3D supervision and identity normalization, are introduced to improve the accuracy. The framework is shown in Fig. 1 and the details are described as follows.

\subsection{Facial Image Model}

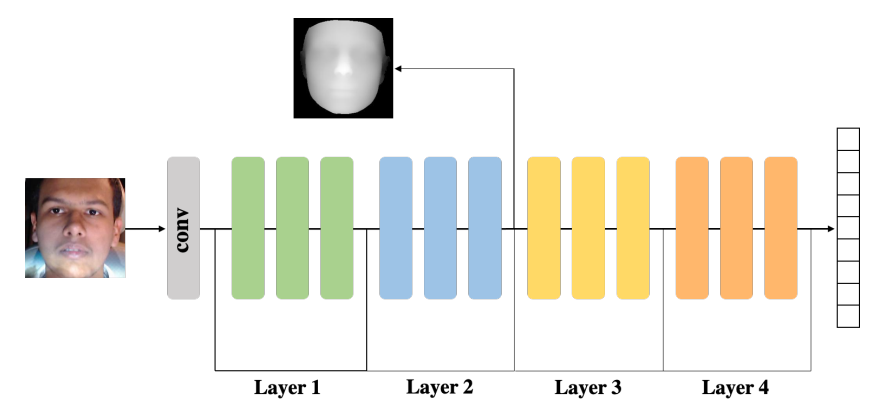

Figure 2: The network architecture of ResNeSt-50 with 3D supervision.

With the great computation ability, deep learning methods have been widely applied in computer vision tasks. Convolutional Neural Networks(CNNs) have been proved to show extraordinary feature representation and transfer ability in the fields of image classification, object detection and recognition. There are so many excellent backbones for the convolutional neural networks. Here, we select ResNet-50[9], ResNeSt-50[21] and EfficientNet[15] to extract the facial feature and use Softmax loss to supervise.

Previous studies have reported that the deeper the convolutional neural networks are, the better representation can be extracted. But with the network depth increasing, the gradients may be vanished or explode and the accuracy will decrease rapidly. To address these problems, He et al. [9] proposed the deep residual network called 'ResNet'. The features can be learned easily with the help of the short connection and the identity mapping will keep the accuracy constant. The gradients can also be transferred in the short connection and the gradients vanish will disappear.

To improve the backbone accuracy and speed, network depth, network width and image resolution are often considered to be the three key points. Tan et al. [15] balance the relationship among the three elements and proposed a compound scaling method. Learned from MnasNet[14], EfficientNet-B0 architecture is defined 
and EfficientNet-B1 EfficientNet-B7 are optimized with the grid search for the hyper parameters. Here, we use EfficientNet-B5 as our backbone to extract facial features.

Due to the limited receptive filed size and the lack of interaction between channels, ResNet-50 may not be the best backbone for certain computer vision tasks. To improve the cross-channel representation, Zhang et al. [21] proposed a backbone called 'ResNeSt'. Compared to ResNeXt proposed by Xie et al. [20], ResNeSt divide the input into k cardinals. In each cardinal, the inputs are splited into $r$ parts and the cardinal group representation is aggregated using channel-wise weighted combination.

In the DGW task, we also introduce 3D information to supervise, which drives the ResNeSt backbone to learning more spatial information. The network architecture can be seen in Fig.2. We know that head pose prediction is related to gaze prediction and with the help of learning head pose information, the network can learn better representation in the gaze task. Inspired by this, we extract the 3D facial mask by PRNet, which proposed by Feng et al. [5] to represent the 3D facial spatial architecture. The feature map from the second layer in ResNeSt-50 is extracted and we compute the element-wise average loss between the feature map and the $3 \mathrm{D}$ depth. The 3D loss will be added to the cross-entropy loss by weight $\lambda$ :

$$
L=L_{c l s}\left(p_{i}, p_{i}^{*}\right)+\lambda \cdot L_{3 D}\left(f_{i}, f_{i}^{*}\right)
$$

Here, $L_{c l s}\left(p_{i}, p_{i}^{*}\right)$ is the Softmax loss, where $p_{i}$ is the predicted probability of sample $i$ and $p_{i}^{*}$ is the ground truth class of sample $i$. $L_{3 D}\left(f_{i}, f_{i}^{*}\right)$ is the mean square error for the feature maps, where $f_{i}$ is the feature map of the sample $i$ from the ResNeSt Layer 2 and $f_{i}^{*}$ is the depth images of the sample $i$ generated from PRNet. We set $\lambda=2500$ according to our experiment to enhance 3D loss effect.

In addition, Qquab et al. [13] showed that image representation learned with CNNs on large-scale annotated datasets can be transferred to other vision tasks easily. Therefore, we use the ResNet-50, EfficientNet-B5 and ResNeSt models which pre-trained on ImageNet dataset. As for the ResNet-50 and ResNeSt-50 model, we froze the first convolutional layer. We modify the output dimension of the last fully connected layer according to the DGW task and fine-tune the above three networks on the DGW dataset.

\subsection{Eyes Image Model}

Recent evidence suggests that the eyes feature plays an important role in gaze prediction task. Vora et al. [19] conducted many experiments about which input pre-processing strategy can do well in driver gaze zone estimation. The results show that upper half of the face get the best accuracy among other input images and the network focus a great attention on double eyes of the drivers. Inspired by this, we extract the 98 landmarks from the faces and cropped the eyes region according to the eyes landmarks. Then, faces, double eyes and single eye are input into ResNeXt-50[20]. We also extract 16 outer contour points of the two eyes and calculated the distance between them and the two pupils. Each distance will be divided by the distance between inner corners, which we define as $d$. There are three branches in eyes model and the left eye and the right eye networks share the same weights. The three branches features will be concated at the feature layer with the normalized distance and sent to the final fully connected layer to output the

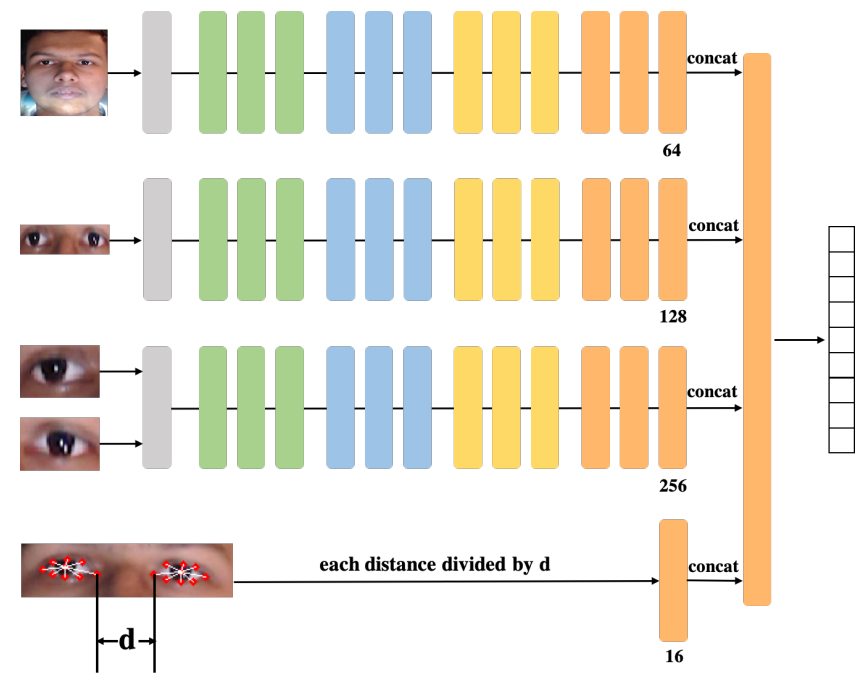

Figure 3: The network architecture of eyes model.

probability of each class with Softmax loss. Overview of the eyes model is shown in Fig.3. The feature dimension of each branch is $64,128,256,16$ respectively in a top-down manner. The cropped eyes images prompt the network to focus more attention on the eyes region and to some extent, the local feature learning is able to lower identity bias among datasets.

\subsection{Landmark Model}

Many of early works use the geometrical representations, including geometry distance and other geometry properties on facial related tasks. Though they may not be better than the CNN-based methods in certain tasks, geometrical representation still works and provides us with a unique kind of feature. Facial landmark is a good geometrical representation and it contains much spatial information.

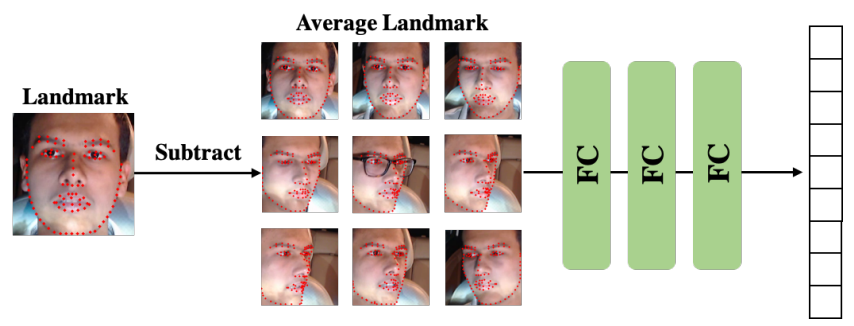

Figure 4: The network architecture of landmark model.

We first extract the 98 landmarks from each aligned face and use the facial landmark represented as a vector, and feed it into the neural network with some fully connected layers. But the performance is not as good as expected. Considering the DGW training and validation data is person-independent, to input the facial landmarks only may cause person bias. In order to reduce the landmarks' variance of the same class in different faces, we adapt a normalization method which each 98-dimensional landmark subtracts an average 
landmark which belongs to the same subject. As for the subject's average landmark, we first calculate this person's average landmark in every class.

$$
\overline{L^{(c)}}=\frac{1}{N} \sum_{i} L_{i}^{(c)}
$$

where $\overline{L^{(c)}}$ is the current person's average landmark on class $c(c=1,2, \ldots, 9)$ and $L_{i}^{(c)}$ is the current person's $i$ th landmark sample that belongs to class $c$. Then, we calculate the average landmark from the nine classes as follows and define it as this person's average landmark.

$$
\bar{L}=\frac{1}{C} \sum_{c} \overline{L^{(c)}}
$$

For each facial landmark $L$, we will subtract it by the person's average landmark $L_{i n}=L-\bar{L}$ and then feed the vector $L_{i n}$ into the FC layers. Fig. 4 demonstrates the landmark model framework. The experiment shows that the result is better than the non-normalized method and the person bias can be reduced greatly.

The premise of calculating the average landmark is to know the identity of this face and which class it belongs to. In the DGW task, the identity is available from the picture name in training and validation data. As for the test data, we use a LResNet100E-IR face recognition model trained by MS1M dataset with Arcface[2] loss and then extract each aligned face's 512-demensional feature. With these face features, we conduct face clustering and get every image's identity in test data. Apart from the identity, class information is also required in the test data. Here we use the fusion prediction from the three facial models and the eyes model as the test data pseudolabel. With the identity and the pseudo-label, we can calculate the average landmark in test data.

\subsection{Delaunay Model}
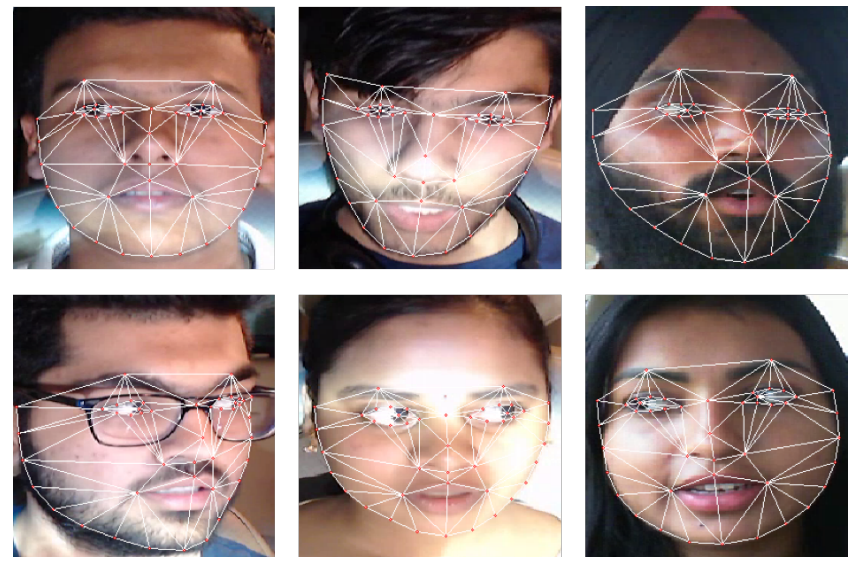

Figure 5: Some face samples under our Delaunay triangulation.

Apart from the landmark representation, there are other geometrical representations which can supplement better geometric features. Fridman et al. [6] extract 19 points from the facial landmark and design a Delaunay triangulation over these 19 points. The three angles of each of the resulting triangles are computed and added to the landmark vector together. Inspired by the Delaunay triangulation, we select 45 points from the 98 landmarks based on the generality of faces and design a Delaunay triangulation over these 45 points based on the face modeling approach in [8]. The Delaunay triangulation is kept same for all images and we get 69 triangles resulting 207 angles. Our Delaunay triangulation samples over these 45 points are shown in Fig.5. Similar to the landmark model preprocessing, each 207 angles also subtract the person's average Delaunay angles and then feed the vectors into the FC layers to output classification probabilities. The calculation method of average Delaunay angles is same to the average landmark in landmark model by the Equation. 2 and 3.

\subsection{Fusion Strategy}

In previous sections, we present different models in different feature representations. Each of these models can extract unique features and make more or less contribution to the final prediction. Therefore, we have to design a strategy to fusion the output of each network. In our framework, we extract a score vector for each sample which means the probability of the sample belongs to the specific class. We use weighted sum in different models and get the final prediction as below.

$$
S=\sum_{i} w_{i} \cdot S_{i}
$$

Where $S$ is the final score vector and $w_{i}$ and $S_{i}$ are the weight and score vector of network i respectively. We use grid search method on validation data to find the best weights for the six models above. In order to get better performance, we horizontal flip the images but keep the label same and train another six models with the flipped data. The final weight is $0.77,0.87,0.51,0.0,0.64,0.72,0.63,0.94,0.44,0.17$, $0.44,0.71$ for ResNeSt-50, landmark model, delaunay model, eyes model, EfficientNet-B5, ResNet-50 and their flipped models respectively.

\section{EXPERIMENT}

\subsection{Implementation Details}

Pre-Processing. In order to eliminate the influence of pose, face detection and alignment are usually necessary in face-related tasks. In the DGW task, we use RetinaFace[3] and DFS [17] [22] to detect faces and their five landmarks. Then, we align the faces according to the extracted landmarks and resize the faces to size $256 \times 256$. In our facial model, different size of the aligned faces are input into different networks. $320 \times 320,480 \times 480,224 \times 224$ size of faces are used for ResNeSt-3D, EfficientNet-B5 and ResNet-50 respectively. In our eyes model, we use cropped $96 \times 96$ size of faces without alignment as one of the input branch. After the 98 landmarks extraction, we cropped the double eyes and single eye according to the eyes landmarks and then cropped them into $192 \times 64$ and $92 \times 64$ respectively. Because of the different size of receptive fields in different networks, we decide to use different input images size to make our fusion more effective. Moreover, increasing image resolution 
can help model to learn more detail features, especially in the gaze prediction task which should pay more attention to eyes' details.

As for the ResNeSt-50 network with 3D information, we need to get the corresponding 3D depth image from each aligned face. There are many brilliant works on 3D face reconstruction and here we use PRNet[5] to extract 3D depth face images in $\mathrm{z}$ direction. After that, we resize the depth images into size $40 \times 40$ for the output feature map of layer two in ResNeSt-50. Some samples for the 3D depth images are shown in Fig.6.
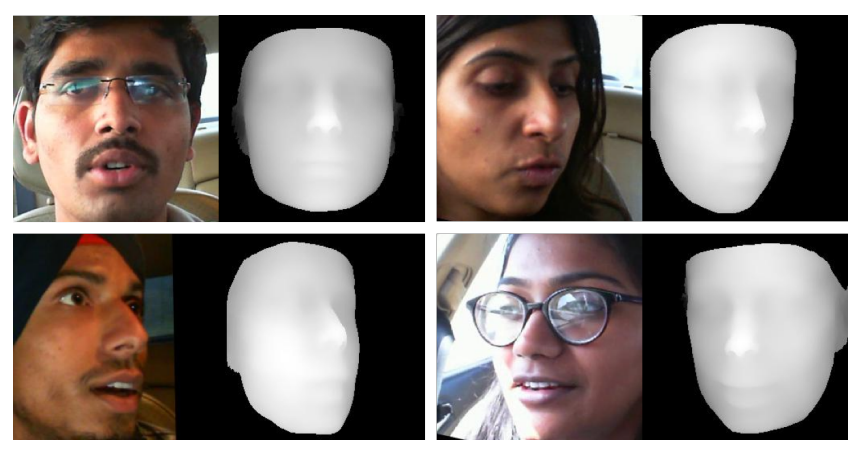

Figure 6: Some aligned face samples and their depth images generated by PRNet.

After the face detection and alignment, we need to get the 98 landmarks from the aligned faces for the landmark model and Delaunay model. PropagationNet [10], proposed by Huang et al., is a heatmap regression model for facial landmark detection, which propagates landmark heatmaps to boundary heatmaps to better facilitate the learning of structural information. We use the model in Huang's work to extract 98 landmarks for our landmark model and Delaunay model. The 98-dimensional landmarks are flattened into 196-demensional vector for each face and then feed it into the FC layers.

Training Details. In our experiment, we use Pytorch deep learning framework and different models have different hyper parameters. We set batch size as 64, 32, 64 and learning rate as 1e-2, 1e-3, 5e-3 in ResNeSt-50 model, EfficientNet-B5 model and ResNet-50 model respectively and train for 60 epochs. As for ResNeSt-50 and ResNet-50 models, we froze the first convolutional layer for better transfer learning. We use SGD optimizer in ResNeSt-50 and ResNet50 models, and Adam for EfficientNet-B5 model. Cosine annealing learning rate decay strategy is used in the three facial models. As for eyes models, batch size and learning rate are 64 and 2e-3 and we train 50 epochs using Adam optimizer. All the image-related models are loaded with ImageNet pre-training weights.

For training landmark model and Delaunay model, we use optuna, a hyperparameter optimization framework, to search the best hyperparameter for FC dimension, dropout parameter, learning rate and optimizer. After many iterations, the landmark model FC layers are $215,219,312,442$ and trained by Adam optimizer with $7 \mathrm{e}-4$ learning rate. And for Delaunay model, FC layers are 468, 503, 509 and trained by SGD optimizer with $6 \mathrm{e}-3$ learning rate. All the models we proposed will be supervised by Softmax loss, which in order to reduce intra-class distance and increase inter-class distance.
Data Augmentation. Data augmentation is an effective method to reduce overfitting and improve the generalization ability of the model. Equalizing the images histogram is commonly used to reduce the influence of illumination. In the DGW dataset, images are collected during different times of the day which result in a huge illumination variance. So, we equalize the histogram of each faces with the probability of 0.5 . We have tried to horizontal flip or rotate the images but it leads to a significant drop in the accuracy.

To make our fusion more effective, we horizontal flip all the faces in training, validation and test data but keep the label same. We train all the models again with these flipped images and get double predictions for the final fusion. The flipped images are in a new feature space compared to the original images and the models may learn a different feature representation.

In order to learning from more data, we divide the validation data into two parts based on the person identity. We put half of the validation data into the training data and the others remain as validation data. Therefore, we get two new sets of training and validation data. Similar to the original set of data, we train another two sets of models with the two sets of dataset. The predictions from the three sets of models are fused together and achieve $82.5288 \%$ on the test database.

\subsection{Impact of Weight in 3D Supervision}

In ResNeSt-50 model, we introduced 3D supervision to enable the network to better learn spatial features. At the same time, we use $\lambda$ to balance the weight relationship between 3D loss and classification loss. The experiment results for the 3D supervision effectiveness and the influence of different weight $\lambda$ on final model accuracy are shown in the table below.

Table 1: Impact of weight $\lambda$ on the model accuracy.

\begin{tabular}{cccccccc}
\hline$\lambda$ & 0 & 500 & 1000 & 1500 & 2000 & 2100 & 2200 \\
$\operatorname{Acc}(\%)$ & 76.75 & 76.36 & 76.48 & 75.54 & 76.00 & 76.55 & 76.85 \\
\hline \hline$\lambda$ & 2300 & 2400 & 2500 & 2600 & 2700 & 2800 & 2900 \\
$\operatorname{Acc}(\%)$ & 76.12 & 76.24 & $\mathbf{7 7 . 3 4}$ & 76.77 & 76.54 & 76.86 & 76.67 \\
\hline
\end{tabular}

We can learn from the Table. 1 that introducing 3D supervision can improve classification accuracy by $0.6 \%$ compared to the baseline $(\lambda=0)$. Meanwhile, the weight of 3D loss has an impact on the final accuracy and we set $\lambda=2500$ to get better model.

\subsection{Impact of Normalization in Landmark and Delaunay Model}

Although we have aligned the faces according to the five landmarks, the landmarks and Delaunay angles still have a big variance due to the subjects and camera placement. Therefore, we consider normalizing the landmarks and Delaunay angles to reduce the identity bias. We have tried two normalization methods and the effects are demonstrated below.

Person Mean method indicates that all the landmarks belong to the same person are summed, regardless of the class information, and get the average landmark for this person ID. Class Mean method means that, for the current person ID, each class's average landmark is calculated separately by Equation.2, and then calculate the class 
Table 2: Comparison of different normalization methods.

\begin{tabular}{cccc}
\hline Model & $\begin{array}{c}\text { Non-Normalization } \\
(\%)\end{array}$ & $\begin{array}{c}\text { Person Mean } \\
(\%)\end{array}$ & $\begin{array}{c}\text { Class Mean } \\
(\%)\end{array}$ \\
\hline Landmark & 47.83 & 67.15 & $\mathbf{7 4 . 3 8}$ \\
Delaunay & 60.47 & 63.62 & $\mathbf{7 1 . 5 0}$ \\
\hline
\end{tabular}

average landmark by Equation.3. We can see from the Table.2 that Class Mean method shows better performance than Person Mean method and non-normalization method. We guess that Class Mean method can reduce the impact of inter-class noise and make the final average landmarks or Delaunay angles more stable.

\subsection{Pseudo Label Method}

We have confirmed that with normalized landmarks or Delaunay angles, the classification accuracy can be improved greatly. As mentioned in the previous section, identity information and category information are important prerequisites for normalized landmarks or Delaunay angles.

Table 3: Impact of pseudo label on the validation data.

\begin{tabular}{ccc}
\hline Model & True Label(\%) & Pseudo Label(\%) \\
\hline Landmark & 74.38 & 73.67 \\
Delaunay & 71.50 & 70.29 \\
\hline
\end{tabular}

For access to identity information, we can get it from the picture names in training and validation data. As for test data, we extract the face features by a face verification model which is a LResNet100EIR network trained by MS1M and Arcface[2] loss, and finish the face clustering with face features. When obtaining the category information of the test data, we adapt a pseudo label method. We fusion the three facial models, including ResNeSt-50, EfficientNetB5, ResNet-50, and the eyes model. We find the best fusion weights by grid search optimization on validation set and make the pseudo label for the validation and test data in landmark and Delaunay models. We evaluate the impact of pseudo label on the final results and the results are shown in the table below.

From the Table.3, the accuracy of the pseudo label method is lower than the true label method, but the decline degree is quite acceptable for us. It is still higher than the non-normalized method and we can infer that there will not be a significant drop in test data.

\subsection{Identity Normalization}

As described in the previous sections, subject bias is a key point in the DGW task and we utilize different identity normalization methods to enhance the generalization ability of our models. The effectiveness of our identity normalization methods are demonstrated below.

From the Fig.7, we can make a conclusion that various models we proposed can be optimized by different kinds of identity normalization methods. 3D supervision, facilitating facial model to learn head pose implicitly, can slightly increase the accuracy by about $0.6 \%$. But in the landmark and Delaunay model, subtracting the average landmark or Delaunay angles of the current person

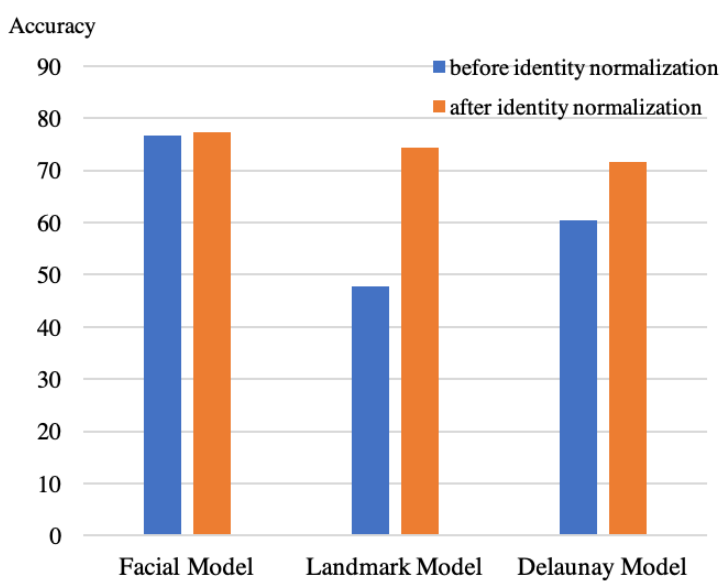

Figure 7: Effect of identity normalization.

can obtain a dramatic improvement, with $26.5 \%$ and $11.0 \%$ up in accuracy respectively. It is surprised to find that the geometric representation can get comparable performance compared to appearance representation, which make a better foundation for the final model fusion.

\subsection{Ablation Study}

In order to study the influence of different models on the final fusion result, we tried different combinations of model fusion, and the results are shown in the Table 4 . It can be seen from the table that if we only consider the appearance features and ignore the geometric features(i.e. Landmark Model and Delaunay Model), we can't get the best performance. However, the representation ability of geometric features is also very limited, so ignoring visual features will also bring huge performance degradation. Last but not the least, fusion with eyes model can slightly improve the accuracy of gaze prediction. This kind of local feature cannot be ignored.

Table 4: The Influence of Different Models on Fusion Results

\begin{tabular}{ccccc}
\hline Models & \multicolumn{3}{l}{ Whether to fusion or not } \\
\hline ResNeSt-50 & $\checkmark$ & $\checkmark$ & $\checkmark$ \\
EfficientNet-B5 & $\checkmark$ & $\checkmark$ & $\checkmark$ \\
ResNet-50 & $\checkmark$ & $\checkmark$ & & $\checkmark$ \\
Eyes Model & $\checkmark$ & & & $\checkmark$ \\
Landmark Model & & $\checkmark$ & $\checkmark$ & $\checkmark$ \\
Delaunay Model & & $\checkmark$ & $\checkmark$ & $\checkmark$ \\
\hline Fusion Accuracy on Validation & 79.13 & 80.24 & 75.68 & $\mathbf{8 0 . 2 9}$ \\
\hline
\end{tabular}

\subsection{Results and Discussion}

The results of each model and its flipped model in different datasets are shown in Table.5. It can be seen from the table that ResNeSt50 with 3D supervision get the best result among all the models due to its advantageous network structure and 3D supervision algorithm. As we all know, EfficientNet-B5 and ResNet-50 are also 
Table 5: Classification accuracy of each model on the validation and test dataset.

\begin{tabular}{|c|c|c|c|c|c|}
\hline \multirow{2}{*}{ Models } & \multirow{2}{*}{ Input Type } & \multicolumn{3}{|c|}{ Validation } & \multirow{2}{*}{ Test } \\
\hline & & Original Dataset & Split-1 Dataset & Split-2 Dataset & \\
\hline \multirow{2}{*}{ ResNeSt-50 } & src & 77.34 & 78.14 & 79.17 & - \\
\hline & flip & 76.23 & 78.66 & 78.51 & - \\
\hline \multirow{2}{*}{ EfficientNet-B5 } & src & 75.11 & 77.82 & 77.55 & - \\
\hline & flip & 74.76 & 77.43 & 77.73 & - \\
\hline \multirow{2}{*}{ ResNet-50 } & src & 74.55 & 75.77 & 76.65 & - \\
\hline & flip & 73.42 & 76.51 & 75.50 & - \\
\hline \multirow{2}{*}{ Eyes Model } & src & 66.77 & 69.10 & 69.90 & - \\
\hline & flip & 67.26 & 70.16 & 68.73 & - \\
\hline \multirow{2}{*}{ Landmark Model } & src & 73.67 & 74.97 & 74.00 & - \\
\hline & flip & 73.77 & 74.31 & 74.31 & - \\
\hline \multirow{2}{*}{ Delaunay Model } & src & 70.29 & 70.57 & 72.09 & - \\
\hline & flip & 70.15 & 71.06 & 71.77 & - \\
\hline Fusion & & 80.29 & 83.15 & 82.06 & 82.5288 \\
\hline
\end{tabular}

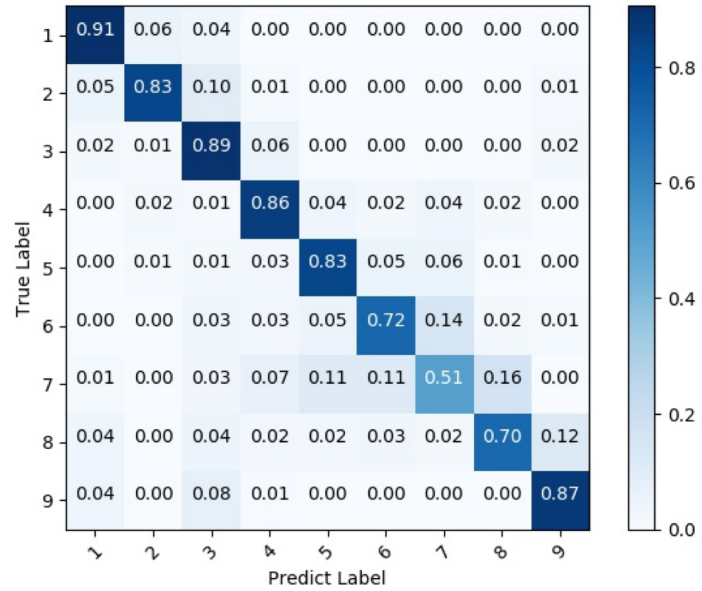

Figure 8: Confusion matrix of the original validation dataset after fusion.

excellent backbone networks and they can achieve equivalent classification performance to ResNeSt-50 with 3D supervision. Through our experiments, we found that global facial features are the most discriminative features.

By comparing the eye model and three appearance models, we can find that more information allows the model to learn more features, and therefore global features are more effective than local features. Although the accuracy of eyes model is lower than other models, the final fusion is quite effective and the accuracy of model fusion is about $3 \%$ higher than the best sub-model.

Finally, the two geometric features we proposed have also achieved comparable performance to visual features. After our proposed identity normalization preprocessing operation, these geometric features also have strong representation capabilities.

In addition, dividing the original dataset into two sets and fusion the three parts together can also improve the accuracy slightly. The final submission achieves $82.5288 \%$ accuracy on the test data and ranks 1st in the driver gaze estimation sub-challenge at EmotiW2020.

The confusion matrix of fusion networks is shown in Fig.8. We can make a conclusion that Label 6, Label 7 and Label 8 are hard to classify correctly, perhaps because the label from six to eight are adjacent zones separated by subtle eye movement. How to improve the model's ability to distinguish these adjacent areas is a key point in the future work.

\section{CONCLUSION}

In this paper, we propose a multi-model fusion method to classify the driver gaze at the EmotiW2020. We use three state-of-the-art $\mathrm{CNNs}$, i.e. ResNeSt-50, EfficientNet-B5, ResNet-50 to extract facial features. In eyes network, we adapt three branches fusion strategy to learn the eyes representation from faces, double eyes and single eye. Apart from the visual representations, we also obtain geometric features from the landmarks and Delaunay angles models. 3D supervision is applied to help the network learning better spatial information and normalization with pseudo label method is designed to enhance geometric feature representation. With these identity normalization methods above, we can get better performance in this person-independent task. In the end, we use grid search to find the best weights for the final fusion. The submission result is $82.5288 \%$ on the test dataset, which ranks 1st at the EmotiW2020 driver gaze prediction sub-challenge.

\section{ACKNOWLEDGMENTS}

This work was sponsored by DiDi GAIA Research Collaboration Initiative. 


\section{REFERENCES}

[1] Zhaokang Chen and Bertram Shi. 2020. Offset Calibration for Appearance-Based Gaze Estimation via Gaze Decomposition. In The IEEE Winter Conference on Applications of Computer Vision. 270-279.

[2] Jiankang Deng, Jia Guo, Niannan Xue, and Stefanos Zafeiriou. 2018. ArcFace: Additive Angular Margin Loss for Deep Face Recognition. arXiv: Computer Vision and Pattern Recognition (2018).

[3] Jiankang Deng, Jia Guo, Yuxiang Zhou, Jinke Yu, Irene Kotsia, and Stefanos Zafeiriou. 2019. RetinaFace: Single-stage Dense Face Localisation in the Wild. arXiv: Computer Vision and Pattern Recognition (2019).

[4] Abhinav Dhall, Garima Sharma, Roland Goecke, and Tom Gedeon. 2020. EmotiW 2020: Driver Gaze, Group Emotion, Student Engagement and Physiological Signal based Challenges. ACM International Conference on Multimodal Interaction 2020 (2020).

[5] Yao Feng, Fan Wu, Xiaohu Shao, Yanfeng Wang, and Xi Zhou. 2018. Joint 3d face reconstruction and dense alignment with position map regression network. In Proceedings of the European Conference on Computer Vision (ECCV). 534-551.

[6] Lex Fridman, Philipp Langhans, Joonbum Lee, and Bryan Reimer. 2015. Driver Gaze Region Estimation Without Using Eye Movement. arXiv: Computer Vision and Pattern Recognition (2015).

[7] Shreya Ghosh, Abhinav Dhall, Garima Sharma, Sarthak Gupta, and Nicu Sebe. 2020. Speak2Label: Using Domain Knowledge for Creating a Large Scale Driver Gaze Zone Estimation Dataset. arXiv:arXiv:2004.05973

[8] Reza Hassanpour and Volkan Atalay. 2004. Delaunay Triangulation based 3D Human Face Modeling from Uncalibrated Images. (2004), 75-75.

[9] Kaiming He, Xiangyu Zhang, Shaoqing Ren, and Jian Sun. 2016. Deep Residual Learning for Image Recognition. (2016), 770-778.

[10] Xiehe Huang, Weihong Deng, Haifeng Shen, Xiubao Zhang, and Jieping Ye. 2020 PropagationNet: Propagate Points to Curve to Learn Structure Information. In Proceedings of the IEEE/CVF Conference on Computer Vision and Pattern Recognition. 7265-7274.

[11] Sumit Jha and Carlos Busso. 2018. Probabilistic Estimation of the Gaze Region of the Driver using Dense Classification. (2018), 697-702.
[12] Rizwan Ali Naqvi, Muhammad Arsalan, Ganbayar Batchuluun, Hyo Sik Yoon, and Kang Ryoung Park. 2018. Deep Learning-Based Gaze Detection System for Automobile Drivers Using a NIR Camera Sensor. Sensors 18, 2 (2018), 456.

[13] Maxime Oquab, Leon Bottou, Ivan Laptev, and Josef Sivic. 2014. Learning and Transferring Mid-level Image Representations Using Convolutional Neural Networks. (2014), 1717-1724.

[14] Mingxing Tan, Bo Chen, Ruoming Pang, Vijay K Vasudevan, Mark Sandler, Andrew Howard, and Quoc V Le. 2018. MnasNet: Platform-Aware Neural Architecture Search for Mobile. arXiv: Computer Vision and Pattern Recognition (2018).

[15] Mingxing Tan and Quoc V Le. 2019. EfficientNet: Rethinking Model Scaling for Convolutional Neural Networks. (2019), 6105-6114.

[16] Ashish Tawari and Mohan M Trivedi. 2014. Robust and continuous estimation of driver gaze zone by dynamic analysis of multiple face videos. (2014), 344-349.

[17] Wanxin Tian, Zixuan Wang, Haifeng Shen, Weihong Deng, Binghui Chen, and Xiubao Zhang. 2018. Learning Better Features for Face Detection with Feature Fusion and Segmentation Supervision. arXiv: Computer Vision and Pattern Recognition (2018).

[18] Borhan Vasli, Sujitha Martin, and Mohan M Trivedi. 2016. On driver gaze estimation: Explorations and fusion of geometric and data driven approaches. (2016), 655-660.

[19] Sourabh Vora, Akshay Rangesh, and Mohan M Trivedi. 2018. Driver Gaze Zone Estimation using Convolutional Neural Networks: A General Framework and Ablative Analysis. arXiv: Computer Vision and Pattern Recognition (2018).

[20] Saining Xie, Ross Girshick, Piotr Dollar, Zhuowen Tu, and Kaiming He. 2017. Aggregated Residual Transformations for Deep Neural Networks. (2017), 59875995.

[21] Hang Zhang, Chongruo Wu, Zhongyue Zhang, Yi Zhu, Zhi Zhang, Haibin Lin, Yue Sun, Tong He, Jonas Mueller, R. Manmatha, Mu Li, and Alexander Smola. 2020. ResNeSt: Split-Attention Networks. arXiv:arXiv:2004.08955

[22] Xiubao Zhang, Ziyuan Lin, and Wanxin Tian. 2020. Mask-wearing recognition in the wild. Sci Sin Inform, 2020, doi:10.1360/SSI-2020-0046 (2020).

[23] Xucong Zhang, Yusuke Sugano, Mario Fritz, and Andreas Bulling. 2015. Appearance-based gaze estimation in the wild. In Proceedings of the IEEE conference on computer vision and pattern recognition. 4511-4520. 